\section{A PROSPECTIVE STUDY ABOUT THE SURGICAL MANAGEMENT OF THYROID CARCINOMA}

KEY WORDS: Thyroid

Carcinoma , Prospective Study Thyroidectomy

\section{Dr. Anil Kumar Dubey \\ Dr Yuvraj Churendra*}

Asso Professor, Dept Of General Surgery, Raipur Institute Of Medical Sciences, Raipur, $\mathrm{Cg}$, India

Asst Professor, Dept Of General Surgery, Raipur Institute Of Medical Sciences, Raipur , Cg , India *Corresponding Author

INTRODUCTION : Thyroid cancer is one of the most common endocrine cancer and has the highest mortality among endocrine neoplasms. In India, its incidence is between $0.8-2$ per 1,00,000 population. Early diagnosis and treatment remain the cornerstone of management to decrease the mortality rates. lack of prospective randomized trials, the management of these cancers generates tremendous controversy. study was planned to assess \& evaluate the management of various thyroid carcinomas.

METHODS: A total of 40 patients were selected which were pathologically / histologically proven cases of Thyroid carcinoma in this Prospective study design in 2.5 years. Detailed Clinical Examination was done \& Prior to surgery all the patients went through investigations. Subjects included both the genders, all age groups including pediatric and geriatric age group and all classes of socio economic strata.

RESULTS: No of female patients were 30 and male patients 10. Male : female ratio in this study was 1:3. FNAC / Biopsy / Cytology detected papillary carcinoma in 26 patients (65\%), follicular neoplasm in 11 patients (27.5\%), $2(5 \%)$ patients of anaplastic and one $(2.5 \%)$ of medullary carcinoma. $86.6 \%$ of the patients had a firm gland \& hard gland and rest presented with a soft gland on palpation. Total Thyroidectomy with cervical block dissection was performed in $32(80 \%)$ cases . Rest of the $20 \%$ were dealt with Sub total Thyroidectomy with cervical block dissection.

CONCLUSION: Most of the cancers present as a slow growing goiter for few months to few years duration. Most of the cancers are of papillary carcinoma type, FNAC detects the majority of the cancers and is an important diagnostic test. Thyroid cancers have a good prognosis as most of the patients present as Stage I disease \& most of the patients require total thyroidectomy with or without lymph node dissection followed by radio iodine ablation as treatment. Thyroid cancer, irrespective of the extent of disease, has good prognosis

\section{INTRODUCTION}

Thyroid cancer is one of the most common endocrine cancer . Over a decade, the incidence rate of thyroid cancer in India in women increased from 2.4 (95\% confidence interval (CI) 2.2$2.7)$ to $3.9(95 \%$ CI $3.6-4.2)$ and in men from 0.9 (95\% CI 0.8$1.1)$ to $1.3(95 \%$ CI $1.2-1.5)$, a relative increase of $62 \%$ and $48 \%$ respectively ${ }^{1} \&$ it is on the rise due to the increase in detection of asymptomatic nodules by imaging modalities like ultrasonography, computerized tomography (CT) scan and positron emission tomography (PET) scan done for some unrelated indication along with Fine needle aspiration Cytology (FNAC)

The cumulative life-time risk of thyroid cancer is estimated to be one in 970 in males and one in 565 in females ${ }^{2}$

Thyroid cancers cover a large spectrum of disease with diametrically opposite prognosis. At one end of the spectrum we have the well differentiated cancers which carry an excellent prognosis, while at the other end there is anaplastic cancer with high mortality rates and dismal prognosis. Thyroid cancer represents $<1 \%$ of all malignancies. ${ }^{3}$. Majority are well differentiated with papillary and follicular cancers accounting for approximately $79 \%$ and $13 \%$ respectively. They have a favorable prognosis with survival rates of $90-95 \%$ for papillary carcinoma and $80-85 \%$ for follicular carcinoma. Medullary carcinoma, arising from parafollicular C cells accounts for $3-5 \%$ and carries a less favorable prognosis with a 10 year survival of approximately $65-80 \%$. Anaplastic carcinoma is rare and accounts for $2-5 \%$ with a poor prognosis. ${ }^{4}$

Thyroid diseases are different from other diseases in terms of their ease of diagnosis, accessibility of medical treatment, and the relative visibility that even a small swelling of the thyroid offers to the treating physician. Early diagnosis and treatment remain the cornerstone of management to decrease the mortality rates due to thyroid carcinoma. Effective management includes early diagnosis and effective therapy applied at an early tumor stage when it is most amenable to surgery and I-131 therapy.

Various studies have recommended total or near-total thyroidectomy followed by post-operative I-131 thyroid remnant ablation for most patients with differentiated thyroid carcinoma and almost everyone preferred long-term T4 therapy according to the levels of Thyroid Stimulating Hormone. External radiation and chemotherapy also plays an important role in the treatment of undifferentiated thyroid carcinoma. the intensity of treatment must match the aggressiveness of the cancer. Due to the overall excellent prognosis of majority of well differentiated cancers and the lack of prospective randomized trials, the management of these cancers generates tremendous controversy.

Hence this study was planned to assess \& evaluate the management of various thyroid carcinomas.

\section{METHEDOLOGY}

This Prospective study involved Prior Consent from the patients \& was found to be within ethical standards . Patients admitted in the various centres and other surgical units of tertiary care hospitals in Raipur district including Raipur Institute of Medical Sciences, Raipur as diagnosed cases of Thyroid carcinoma were included in this Prospective study. The operating team / Surgeons were the same in all cases.

A total of 40 patients were selected which were pathologically / histologically proven cases of Thyroid carcinoma during a period of 2.5 years from year 2016 to 2019 . Subjects included both the genders, all age groups including pediatric and geriatric age group and all classes of socio economic strata.

Patients who didn't wanted to be part of the study, Benign

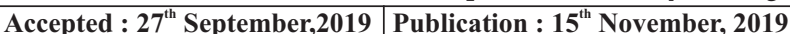


histologically and Doubtful and some false positive cases during Fine needle aspiration Cytology ( FNAC) were excluded from the study .

Detailed Clinical Examination was done \& Prior to surgery all the patients went through investigations , Thyroid profile ( T3, T4, TSH ) , routine blood investigation, Urine examination, Chest X ray , Mammogram (in females ), USG of Neck and adjoining areas , Computed Tomography of Head, Neck \& Thorax.

We followed the ATA 2009 guidelines ${ }^{5}$ and the $7^{\text {th }}$ edition of AJCC TNM staging system. ${ }^{6}$ The FNAC, frozen section and final histopathologic diagnosis were used to decide the extent of the surgery.

Data was filled in Microsoft Excel \& analysed using a computer software Epi Info version 6.2 (Atlanta, Georgia, USA).P value of 0.05 and less was considered as statistically significant.

All the patients underwent surgery after proper consent. Most of the patients were discharged with a clean and healthy wound except a very few with post-operative complications which was duly diagnosed $\&$ treated well in time.

Patients symptomatic for hypocalcemia were classified as those requiring intravenous calcium preparation or oral supplements alone depending on the severity of symptoms along with corrected calcium levels. None of the patients were given calcium preoperatively as a prophylaxis or treated empirically with oral calcium supplements. None of the patients were given any vitamin D supplementation preoperatively. Ionized calcium or parathyroid hormone assay was not done to assess hypocalcemia. All patients were postoperatively assessed for vocal cord mobility.

\section{RESULTS}

Out of the 40 cases with thyroid carcinoma in the study, No of female patients were 30 and male patients 10. Male : female ratio in this study was $1: 3$.

32 of the cases ( $80 \%$ ) of carcinoma thyroid occurred in the age group of 21-50 years, with the average age being 34 years with a minimum of 16 years and a maximum of 70 years. Only 2 cases were seen after 50 years of age.

In all of the patients (100\%) presenting complain was swelling of the thyroid (Single / Multiple Nodular swelling)

$3(7.5 \%)$ of patients had hoarseness of voice, which was the most common pressure effect. Features of hyperthyroidism were present in 4 cases i.e $10 \%$ of patients. Palpable lymph nodes were present in $20 \%$ of cases.

33 of the patients $(82.5 \%)$ presented with symptoms during the past 6 months to 3 years. Only $4.4 \%$ of cases had swelling for $>5$ years. $30 \quad(75 \%)$ of the tumors were slow growing, and $10(25 \%)$ were fast growing tumors.

The present study had $30 \%$ patients presented with tumor size $>4 \mathrm{~cm}, 50 \%$ with nodal metastasis and $10 \%$ had distant metastasis at presentation.

$86.6 \%$ of the patients had a firm gland \& hard gland and rest presented with a soft gland on palpation.

FNAC / Biopsy / Cytology detected papillary carcinoma in 26 patients $(65 \%)$, follicular neoplasm in 11 patients $(27.5 \%), 2$ $(5 \%)$ patients of anaplastic and one $(2.5 \%)$ of medullary carcinoma.

6 patients (15\%) showed unilateral vocal cord palsy. Bony distant metastasis was the most common presentation followed by lung metastases.

28 of the cases (70\%) after investigations and pathological reporting were found to be in Stage I.

Total Thyroidectomy with cervical block dissection was performed in $32(80 \%)$ cases . Rest of the $20 \%$ were dealt with Sub total Thyroidectomy with cervical block dissection.

Patients were observed in post-operative period for any complications. Transient hypoparathyroidism was noted in 10 patients $(25 \%)$. Serum calcium was estimated . 2 Patients were symptomatic hypocalcemic \& were treated with $10 \mathrm{ml}$ of $10 \%$ calcium gluconate intravenously. All patients recovered in the immediate post-operative period. 3 patients had wound infection who improved with antibiotics and dressings. None of the patients had recurrent laryngeal nerve injury postoperatively in our study. No other complications were observed in this study. All the patients with differentiated thyroid cancers were referred to higher centers for radioiodine therapy.Patients who presented with medullary carcinoma were advised a regular follow-up. The patients with anaplastic carcinoma was referred to Cancer Hospital for external beam radiotherapy.

The patients with medullary and anaplastic carcinoma treated with total thyroidectomy were started on replacement doses of thyroxine, whereas those with differentiated thyroid cancer were referred for radioiodine ablation.

\section{DISCUSSION}

Total 40 study subjects were studied. Females are affected more than men, and the ratio was 3:1 in our study. Globally, the annual estimated incidence of thyroid cancer is 298,102 in the year 2012. In India, the incidence is 3960 for men and 9944 for women. The incidence of age-standardized ratio is 0.7 for males (World 1.7) and 1.7 for females (World 6.1). ${ }^{7}$ Overall papillary cancer is more common than follicular which is more common than medullary which is more common that anaplastic cancer. In general, papillary carcinoma peaks in early adult life and then gradually decreases in frequency, whereas the incidence of follicular carcinoma tends to peak sometime later. Anaplastic cancer occurs later in life than differentiated cancers, Similar observations were found in our study. In India, thyroid malignancies constitute $1.2 \%$ and $1.9 \%$ of malignancies in men and women, respectively ${ }^{8}$, The incidence of malignancy in the thyroid nodule was found to be $3-4 \%$, especially the patients with a history of irradiation, family history, male sex, and rapidly growing tumors ${ }^{9}$.

Thyroid cancer most commonly presents as a single neck mass noted incidentally. FNAC is the easiest, least expensive, and most accurate method for diagnosing a thyroid nodule, and their value is universally acknowledged. Ultrasonography using B-mode gray scale provides remarkable anatomic information about the thyroid gland. Because of its simplicity and ability to distinguish between solid and cystic lesions, it is often the first modality to evaluate a thyroid mass in the euthyroid patient. Good quality ultrasound using $7.5-10 \mathrm{MHz}$ transducers provides excellent detail of the superficial gland but requires enough penetration to evaluate posteriorly to the level of the spine. A Mass in elderly is likely to be malignant. An Undifferentiated Thyroid carcinoma is usually fixed to the underlying tissues. Solid lesions have a $21 \%$ risk of malignancy, cystic $7 \%$ and mixed lesions $12 \% .5-10 \%$ of multiple nodules and $10-20 \%$ of solitary nodules are malignant. ${ }^{10,11}$

In Some Carcinomas like papillary carcinoma the presenting feature may be an enlarged Cervical group of Lymph node. The present study had $30 \%$ patients presented with tumor 
size $>4 \mathrm{~cm}, 50 \%$ with nodal metastasis and $10 \%$ had distant metastasis at presentation. Thyroid cancers have the potential to invade the strap muscles, RLN, trachea, esophagus, great vessels, larynx and prevertebral space in that order of frequency. In spite of local invasion, surgery remains the mainstay of treatment to achieve an $\mathrm{R} 0$ resection status even if this means sacrificing surrounding structures.Mayo clinic data reported about $6.1 \%$ RLN infiltration. ${ }^{12}$ The present study had similar rates. Various studies have reported vocal cord palsy rates of $2 \%-15 \% .{ }^{13,14}$ present study had similar rates.

The incidence of postoperative hypothyroidism following thyroidectomy ranges from $21.1 \%$ to $64.2 \%$ in the literature. ${ }^{15}$ Our study recorded $25 \%$. The surgical procedures which are usually advocated by experts are hemithyroidectomy, near-total thyroidectomy, and total thyroidectomy ${ }^{16} \&$ same were used by us.The limitation of this study is that we couldn't able to report long-term result for survival and complications including permanent hypocalcemia. No parathyroid assay was employed for assessment of hypoparathyroidism

\section{CONCLUSION}

Most of the cancers present as a slow growing goiter for few months to few years duration. Most of the cancers are of papillary carcinoma type, FNAC detects the majority of the cancers and is an important diagnostic test. Thyroid cancers have a good prognosis as most of the patients present as Stage I disease \& most of the patients require total thyroidectomy with or without lymph node dissection followed by radio iodine ablation as treatment.

Thyroid cancer, even with advanced disease, is eminently treatable, with surgery being the mainstay of treatment. The complications of thyroid surgery and treatment can be severe for this indolent disease and minimized with meticulous dissection and attention to various details.

\section{ACKNOWLEDGEMENTS}

We would like to thank Professor \& Head, Dept. of Surgery for his always available guidance.

Compliance With Ethical Standards.

\section{Conflict Of Interest -None.}

\section{Funding-None.}

Informed Consent Obtained.

\section{REFERENCES}

1 - Janeesh Sekkath Veedu, Kevin Wang ( 2018) Trends in thyroid cancer Incidence in India. Journal of Clinical Oncology ,Vol 36. el8095-e 18095.

2. Dorairajan N, Pandiarajan R, Yuvaraja S. A descriptive study of papillary thyroid carcinoma in a teaching hospital in Chennai, India. Asian J Surg 2002;25:300-3

3. AACE/AAES Medical Surgical Guidelines for Clinical Practice. Management of thyroid carcinoma. Endocr Pract 2001;7:203-17

4. Devendra A. Chaukar et al. (2010) Management of thyroid cancers, Indian J Surg Oncol 1(2):151-162

5. American Thyroid Association (ATA) Guidelines Taskforce on Thyroid Nodules and Differentiated Thyroid Cancer, Cooper DS, Doherty GM Haugen BR, Kloos RT, Lee SL, et al. Revised American Thyroid Association management guidelines for patients with thyroid nodules and differentiated thyroid cancer.Thyroid 2009;19:1167-214

6. Edge SB. American Joint Committee on Cancer; ACS. AJCC Cancer Staging Manual.7th ed.NewYork:Springer;2009.

7. Ferlay J, Soerjomataram I, Ervik M, Dikshit R, Eser S, Mathers C, et al. GLOBOCAN 2012 v1.0, Cancer Incidence and Mortality Worldwide: IARC Cancer Base No. 11. Lyon, France: International Agency for Research on Cancer; 2013

8. Tata Memorial Hospital. Hospital Cancer Registry. Annual Report 1999. Division of Epidemiology and Biostatistics. Mumbai: Tata Memorial Hospital;2003.p. 19-21.

9. DeGrootLJ.Thethyroidandparathyroidgl ands.In:DeGrootLJ,JamesonJ L, editors. Endocrinology.4th ed., Vol. 43. New York: McGraw Hill Publishing; 2001.p. 1399-435.

10. Brennan M, Bloomer W. Cancer of the endocrine system: The thyroid gland. In: Devita V, Helmen S, Rosenberg S, editors. Cancer: Principles and Practice of Oncology. 6thed.Philadelphia,PA:JB LippincottCo.;2002. p. 111,113 MattoxKL editors SabistonTextBookofSurgery.17th ed. Vol. I. Philadelphia PA:Saunders Publications;2004.p. 947-81.

12. McConahey WM, Hay ID, Woolner LB, van Heerden JA, Taylor WF. Papillary thyroid cancer treated at the mayo clinic, 1946-1970: Initial manifestation, pathologic findings, therapy and outcome. Mayo Clin Proc 1986;61:978

13. Jeannon JP, Orabi AA, Bruch GA, Abdalsalam HA, Simo R. Diagnosis of recurrent laryngeal nerve palsy after thyroidectomy: A systematic review. Int J Clin Pract 2009;63:624-9.

14. Chiang FY, Wang LF, Huang YF, Lee KW, Kuo WR. Recurrent laryngeal nerve palsy after thyroidectomy with routine identification of the recurrent laryngeal nerve. Surgery 2005;137:342-7

15. Cho JS, Shin SH, Song YJ, Kim HK, Park MH,Yoon JH, et al. Is it possible to predict hypothyroidism after thyroid lobectomy through thyrotropin, thyroglobulin, anti-thyroglobulin, and anti-microsomal antibody? J Korean Surg Soc 2011;81:380-6

16. Samuel AW Jr. Total thyroidectomy. Mastery of Surgery. 5th ed., Vol. 34. Philadelphia, PA:Elsevier Publishing;2000.p. 543-78. 